\title{
Modeling Insect-Repellent Use for Chikungunya Disease Prevention Among US-Caribbean Travelers
}

\author{
Oghenekaro Omodior $^{1 *}$, Lori Pennington-Gray ${ }^{2}$, Brijesh Thapa ${ }^{2}$ \\ ${ }^{1}$ Department of Recreation, Park and Tourism Studies, School of Public Health, Indiana University, Bloomington, USA \\ ${ }^{2}$ Department of Tourism, Recreation and Sports Management, University of Florida, Gainesville, USA
}

Corresponding Author: Oghenekaro Omodior, PhD, MPH, Assistant Professor, Department of Recreation, Park and Tourism Studies, School of Public Health, Indiana University, 1025 E. 7th Street, Bloomington, Indiana, 47405, USA. Tel: +1-812-856-7031, Fax: +1-812-855-3998, Email: oomodior@indiana.edu

Received August 30, 2017 Accepted September 23, 2017; Online Published November 4, 2017

\begin{abstract}
Introduction: This study examined the relationship between specific psychosocial variables and the use of insect repellents on skin or clothing as a preventive behavior for Chikungunya fever among US travelers to Caribbean destinations.

Methods: A cross-sectional retrospective online survey method was adopted. US residents who travelled to one of 34 Caribbean destinations within the past 12 months and expressed an awareness of Chikungunya fever were invited to participate in this study. Sociodemographic variables, perceived response efficacy, perceived self-efficacy, perceived Chikungunya severity and susceptibility, and self-reported use of insect repellents were investigated.

Results: Results of direct logistic regression analysis revealed a significant association between higher levels of education and the odds of self-reported use of insect repellent on skin or clothing among study participants. Among the proximal Chikungunya-related variables, hierarchical binary logistic regression revealed a significant association between scores on perceived response efficacy, perceived selfefficacy, and perceived severity of self-reported use of insect repellents. These results support the existence of a hierarchical relationship between the more proximal Chikungunya-related variables and self-reported use of insect repellents as a personal protective measure (PPM).

Conclusion: The findings of the current study have important implications for health communication messaging aimed at reducing the spread of Chikungunya among US travelers to Caribbean destinations. It seems essential to establish the use of insect repellent on skin and clothing as a personal preventive measure against Chikungunya disease within an educational context, framed along the lines of Chikungunya severity, response efficacy, and self-efficacy, for US travelers to destinations with a high risk of exposure to Chikungunya disease-carrying mosquitoes.
\end{abstract}

Keywords: Chikungunya Fever, Personal Protective Measure, Protection Motivation Theory, Caribbean

Citation: Omodior O, Pennington-Gray L, Thapa B. Modeling insect-repellent use for chikungunya disease prevention among US-Caribbean travelers. Int J Travel Med Glob Health. 2017;5(4):125-134. doi:10.15171/ijtmgh.2017.25.

\section{Introduction}

The mosquito-borne disease Chikungunya has been in the news in recent years in association with travel, tourism, and outdoor recreation in several Caribbean destinations. The foremost reasons for this are that the disease is endemic in the Caribbean and travel to disease-endemic regions is the primary risk factor for mosquito exposure and subsequent infection. ${ }^{1,2}$ Secondly, in the presence of vector-competent mosquitoes of the Aedes species, autochthonous transmission has been reported at new destinations, including parts of the United States and its territories, raising concerns of potential epidemics. ${ }^{1,3}$ Prior to 2013 when Chikungunya disease was first reported in the Caribbean island of St. Martin, ${ }^{5}$ an average of 28 cases were reported annually in the United
States among travelers returning from areas known to be epidemic or endemic for Chikungunya disease. ${ }^{6}$ However, beginning in 2014, soon after the disease appeared in Caribbean destinations, the United States saw an increase in Chikungunya cases among travelers returning from the Caribbean and South America, with nearly 3000 travelrelated cases reported nationwide. ${ }^{3}$ Beyond the immediate infection risk to individual US travelers to disease endemic regions is an even wider public health risk resulting from the possibility for local transmission and/or epidemic events due to (1) the absence of pre-existing protective immunity in the general population, (2) a high viral load in the host and infected vectors, and (3) the wide distribution of the Aedes aegypti and Aedes albopictus mosquitoes in the United States,

Copyright $(\subset 2017$ The Author(s). This is an open-access article distributed under the terms of the Creative Commons Attribution License (http:// creativecommons.org/licenses/by/4.0), which permits unrestricted use, distribution, and reproduction in any medium, provided the original work is properly cited. 
especially along the southern, western, and eastern seaboards of the United States., ${ }^{3,7}$ It is not surprising, therefore, that despite the disease being under-reported and underrecognized, about 5000 cases of autochthonous transmission were reported in the US territories of Puerto Rico, US Virgin Islands, and American Samoa. ${ }^{3}$ Autochthonous Chikungunya cases were also reported in Florida in $2014,{ }^{4}$ prompting health authorities in the United States to brace for the wider spread of the disease. ${ }^{8}$

Roche et $\mathrm{al}^{9}$ reported that human behavior associated with Chikungunya awareness and desire for personal protection were key components in the Chikungunya outbreak propagation at Caribbean destinations. In the absence of specific drug treatments or vaccine prevention of Chikungunya disease, the mainstay of Chikungunya disease prevention has been to increase disease awareness and avoid bites from mosquitoes which transmit the virus. ${ }^{10}$ Recommended personal protective measures (PPMs) include avoiding mosquito-infested habitats, wearing appropriate protective clothing, and use of insect repellents. ${ }^{10-12}$ In many situations, especially among international travelers, the use of insect repellents on skin or clothing at the destination may be the only feasible PPM. The bulk of public health research opinion suggests that the use of insect repellents on skin and clothing is effective for Chikungunya disease prevention. ${ }^{13}$ Results of a study of US travelers to one of several Caribbean destinations during the 2014-2015 period revealed that $74 \%$ of participants used insecticides and repellents on skin or clothing when outdoors at the destination. ${ }^{14}$ Among a sample of US travelers to the Dominican Republic, Millman et $\mathrm{al}^{15}$ reported that, while approximately $95 \%$ of participants reportedly used insect repellent, only $30 \%$ applied it multiple times daily. ${ }^{15}$ Lalani et $a{ }^{16}$ reported higher self-reported use of insect repellents on skin and clothing among travelers to regions with a malaria risk (53\%) compared with regions with a Chikungunya/Dengue risk (16\%). Although the use of mosquito repellents on skin and clothing is generally considered effective in preventing Chikungunya, its sociodemographic and behavioral correlates, particularly among US travelers to Caribbean destinations, has remained largely understudied. Dehecq et $\mathrm{al}^{17}$ suggested that the evaluation of Chikungunya transmission risk should be extended beyond traditional entomologic indices to include individual and environmental risk factors.

Given the endemicity of Chikungunya and the high probability of infection at Caribbean destinations, the close proximity of Caribbean destinations to the continental US, and the large number of US travelers to and from these destinations, understanding the individual travelerassociated predictors of use of insect repellents on skin and clothing may have important implications for the prevention of travel-related disease spread, surveillance, and health promotion program planning. The current study purposed to examine the relationship between sociodemographic factors, psychosocial variables, and the use of repellents on skin or clothing among US travelers to Caribbean destinations. The overarching research question for this study is, "Can the use of insect repellents on skin and clothing as a recommended
PPM for Chikungunya disease be predicted on the strength of (a) perceived severity of Chikungunya, $(b)$ perceived vulnerability to Chikungunya, $(c)$ perceived response efficacy of use of insect repellents as a PPM for Chikungunya disease, (d) perceived self-efficacy to use insect repellents as a PPM for Chikungunya disease, and (e) Chikungunya knowledge, after controlling for sociodemographic variables?"

\section{Theoretical Framework}

Rogers ${ }^{18}$ protection motivation theory is an expectancy-value model of preventative health behavior. According to Rogers, ${ }^{18}$ when an individual receives information about an object of risk, a cognitive mediational process is initiated as part of the coping mechanism for dealing with the threat. A broad range of variables, organized along two processes, attempt to explain this cognitive mediational process. The variables within the twin processes of threat and coping appraisal, which together predict the likelihood of adopting the preventative behavior, include perceived severity, perceived vulnerability, perceived response efficacy, and perceived self-efficacy.

Perceived vulnerability refers to the subjective assessment of the risk of developing a specific health problem. ${ }^{19}$ It has been proposed that individuals may: (1) deny any possibility of vulnerability to a disease condition, and therefore be less likely to take preventive action; (2) admit to the "statistical" possibility of its occurrence (This possibility has little reality for them, since they do not really believe the condition will happen to them.), but be less likely to take preventive action; or (3) express their belief of a real possibility of contracting the condition, and therefore be more likely to take preventive action. ${ }^{20}$ Mixed results of the association between perceived vulnerability and health behavior have been reported. ${ }^{19,21,22}$ Chen et al reported a significant association between perceived vulnerability and getting a flu vaccine, ${ }^{23}$ while Hyman et $\mathrm{al}^{24}$ reported no association between perceived vulnerability and mammography utilization.

Perceived severity concerns evaluations of both clinical and social consequences of a disease. ${ }^{19}$ Clinical consequences involve an assessment of whether the disease could lead to death, reduce physical or mental functioning for long periods of time, or cause permanent disability. Broader social and sometimes more complex implications include an assessment of the effects of the disease on job status, family, and other social relationships. ${ }^{19,20}$ Response efficacy is the belief that the recommended coping response is effective (e.g., "Using insect repellent on my skin or clothing is an effective way to prevent Chikungunya disease transmission"). Self-efficacy, on the other hand, is the belief that one is, or is not, capable of successfully performing the recommended coping response/ behavior (e.g." "I have confidence in my ability to apply insect repellents on my skin or clothing"). ${ }^{25}$ The coping response comprises appraisals of response efficacy, self-efficacy, and any perceived "costs" associated with the adoption of the recommended preventive response. Such costs include inconvenience, expense, unpleasantness, difficulty, complexity, side effects, disruption of daily life, and disruption in enjoyment of activity.

According to PMT, ${ }^{18}$ the decision to adopt a recommended 
protective behavior is positive correlated with perceived severity of the threat, perceived vulnerability to the threat, self-efficacy, and coping efficacy, respectively, for the recommended protective behavior; it is negatively correlated with any response costs associated with the behavior. The theory also suggests that an additive model holds within each of the two appraisal processes, such that when components between the two (i.e. threat and coping) processes are combined, second-order interaction effects occur. The assumption is that in the presence of high response- or selfefficacy, perceived severity and/or vulnerability will have a simple main effect on protective behavior. Conversely, when response- or self-efficacy is low, higher levels of perceived severity and/or vulnerability will have either no effect or a boomerang effect on the recommended protective behavior. Given that the output of the appraisal-moderating process is the decision (or intention) to begin or continue the applicable adaptive responses (coping modes), the typical dependent variables in research involving PMT are measures of behavioral intention and behavior. ${ }^{18,26}$

Rogers ${ }^{18}$ recognized the presence of other factors/variables not directly included in PMT, but which may affect the decision to adopt the recommended behaviors indirectly, by affecting threat and coping appraisals. In this regard, Jessor et $\mathrm{al}^{27}$ identified 2 factor categories: proximal and distal factors. The proximal factors are those variables that are threat-related and health-behavior specific; that is, their existence is adaptive and in direct relation to the threat or recommended behavior. They include threat and coping appraisals, knowledge, and perceived cost. Conversely, the distal factors, or distal determinants of protection motivation, are the individual differences in personality characteristics which are distal or not directly related to the threat or health behavior. Being distal, they do not have any direct reference to the adaptive behavior; nor do they have any obvious or immediate implications for health enhancing behavior, although they can serve a risk or protective function. ${ }^{27}$

PMT has been used as a framework to develop and evaluate persuasive communication and as a social cognitive model to predict health behavior. ${ }^{28}$ In a meta-analysis of studies using PMT which represented more than 20 health issues, Floyd et $\mathrm{al}^{26}$ reported that increases in severity of threat, susceptibility/ vulnerability, response efficacy, and self-efficacy significantly facilitated protection motivation and behaviors. On the other hand, decreases in maladaptive response rewards and adaptive costs resulted in significant increases in adaptive intentions and behaviors. Consistent with the PMT model, the results of the review also confirmed that for studies which combined response efficacy and self-efficacy; or threat susceptibility and severity, increases in these combined groups significantly increased adaptive intentions and behaviors. Although most of the effect sizes were in the moderate range, ${ }^{29}$ all the mean effect sizes were statistically significant. Prior to homogeneity correction, the factors showing the strongest impact on protection motivation were self-efficacy, response efficacy, and combined threat susceptibility and severity. The results of this meta-analysis demonstrate that changes in protection motivation and behaviors correspond with the rational components of PMT. ${ }^{26}$

A second meta-analytic review assessing the overall utility of PMT as a predictive model tested associations between all components of the model. ${ }^{30}$ The results revealed: (1) threatand coping-appraisal components of PMT were useful in predicting health-related intentions; (2) PMT was not as useful in predicting future behavior as it was in predicting concurrent behavior; and (3) the coping-appraisal component of PMT had greater predictive validity than the threatappraisal component. ${ }^{30}$ Other studies which investigated the effect of perceived cost on protection motivation have suggested that some target behaviors may carry a greater cost than others. According to Jones and Leary, ${ }^{31}$ when faced with the threat of skin cancer, research participants preferred using sunscreen as opposed to staying out of the sun, both of which are recommended protective behaviors. Additionally, the same behavior may not be perceived as equally effective for countering different outcomes. Wulfert and Wan ${ }^{32}$ reported, for example, that response efficacy was better correlated with using condoms to protect against pregnancy than to protect against AIDS.

Sociodemographic variables constitute the distal non-risk related predictors for "use of insect repellents" in this study, while Chikungunya knowledge, perceived severity, perceived vulnerability, perceived response efficacy, and perceived selfefficacy represent proximal predictors. Linking the proximal predictor variables to the behavior of use of insect repellents is unproblematic given that their very content implicates their relationship. It remains to be seen whether, individually or collectively, they significantly improve our ability to predict use of insect repellents among at-risk populations as a Chikugunya PPM, after taking into consideration the relationship between sociodemographic variables and use of insect repellents. Within the conceptual framework of this study, it is expected that for US travelers to Caribbean regions with an awareness of the threat of Chikungunya disease together with the use of insect repellents on skin and clothing as a recommended PPM, the decision to adopt the recommended PPM is contingent upon: (1) sociodemographic factors on the one hand, and (2) their level of knowledge together with the cognitive appraisal of both threat and coping mechanisms, respectively, on the other hand. It is also expected that respondents are more likely to adopt the recommended PPMs if they believe that the probability of the threat's occurrence (i.e. probability of contracting Chikungunya disease while present at a Caribbean destination) is high. Thus, the following hypotheses are considered:

Hypothesis 1: A significant correlation exists between Chikungunya knowledge, perceived Chikungunya severity, perceived vulnerability to Chikungunya, perceived response efficacy of use of repellents on skin or clothing, and perceived self-efficacy for use of repellents on skin or clothing;

Hypothesis 2: Sociodemographic factors (i.e. age, gender, education, annual household income) are related to (a) perceived severity, $(b)$ vulnerability, $(c)$ perceived response efficacy, and $(d)$ perceived self-efficacy;

Hypothesis 3: Use of insect repellents is related to sociodemographic factors; 
Hypothesis 4: Use of insect repellents is related to (a) Chikungunya knowledge, (b) perceived severity, (c) vulnerability, $(d)$ perceived response efficacy, and $(e)$ perceived self-efficacy, after controlling for sociodemographic variables respectively.

\section{Methods}

A detailed methodological discussion of study population and data collection instrument has been published elsewhere. ${ }^{14}$ The questionnaire was pretested on a representative sample of 39 respondents to ensure clarity, reliability, and validity. To test the hypotheses, data from a subset of study participants who met an additional previous Chikungunya awareness criteria were analyzed for this study. Previous Chikungunya awareness was measured as a dichotomous (Yes, No) variable using the question, "Have you heard about Chikungunya disease before today?" Only those who answered positively were included in this study. This criterion was necessary, given that $\mathrm{PMT},{ }^{18}$ the framework for this study, suggests that respondents would need to be aware of the threat in order to elicit protection motivation. This subset was composed of a total of 196 participants, aged 21 to 56 years (mean $=32.80$ years, $\mathrm{SD}=8.68$, median age $=31.0$ years), including both males $(n=93,50.5 \%)$, and females $(n=91,49.5 \%)$. Twelve participants $(6.0 \%)$ refused to indicate their gender. The sample were predominantly Caucasians $(n=129,69.0 \%)$, followed by African Americans $(\mathrm{n}=22,11.8 \%)$, Asians ( $\mathrm{n}=21$, $10.7 \%)$, and Latino/Latina $(\mathrm{n}=15,8.0 \%)$.

Besides socio-demographic information, all data collected and analyzed were related to key constructs in the study model (Figure 1). These included self-reported use of insect repellents on skin or clothing when outdoors at the primary Caribbean destination as a Chikungunya PPM, Chikungunya knowledge, perceived severity of Chikungunya disease, perceived vulnerability to Chikungunya disease, perceived response efficacy, and perceived self-efficacy.

Exploratory factor analysis (EFA) and item selection were based on the following criteria: (1) Items were subjected to principal component analysis with promax rotation; (2) The Kaiser-Meyer-Olkin measure of sampling adequacy had to be $\geq 0.60^{33}$; (3) Bartlett test of sphericity had to be statistically significant $(P<0.05)$, thus supporting the factorability of the correlation matrix; and 4) The internal reliability of all multiitem constructs was measured using Cronbach $\alpha$. Items were retained and deemed to have good internal consistency if Cronbach a was $\geq 0.70 .{ }^{34}$ Figure 1 represents the conceptual framework based on the EFA loading matrix, Cronbach $\alpha$, and operationalization of study constructs for the hypothesized relationships among predictors of use of insect repellents on skin or clothing.

Personal protective measure: A single item rated on a binary (Yes/No) scale was used to assess use of insecticides and repellents on skin or clothing when outdoors at the primary Caribbean destination. Twelve items adapted from the US Centers for Disease Control and Prevention ${ }^{35}$ were used to assess Chikungunya knowledge. These consisted of 8 True/ False/Don't know items and 4 multiple choice items. Each of the 12 knowledge items was dummy coded as "1" (participant answered the question correctly) or " 0 " (participant answered the question incorrectly). Thereafter, an index of knowledge score measured on a continuous scale was computed.

A single item adapted from by $\mathrm{Ho}^{36}$ was used to assess perceived response efficacy of use of insect repellents on skin or clothing. The item was measured on a 5-point Likert scale $(1=$ strongly disagree to $5=$ strongly agree). Perceived self-efficacy was assessed with 2 items also measured on a 5 -point Likert scale ( $1=$ strongly disagree to $5=$ strongly agree). Four items measured on a 5-point Likert scale ( $1=$ strongly disagree to $5=$ strongly agree) were used to assess perceived severity of Chikungunya disease. ${ }^{37,38}$ The final variable was computed from the mean scores for all 4 items. The mean perceived severity of Chikungunya was 3.7 $(\mathrm{SD}=0.71$, Median =3.75, Minimum $=2, \quad$ Maximum $=5.0$, Skewness $=-0.30, \quad$ Kurtosis $=-0.72, \quad \mathrm{~N}=196) . \quad$ Perceived vulnerability was computed from the mean score of the 2 items which loaded on this factor.

All data was analyzed using IBM SPSS version 24.0.

\section{Results}

The relationship between Chikungunya knowledge, perceived Chikungunya severity, perceived vulnerability to Chikungunya, perceived response efficacy of use of insect

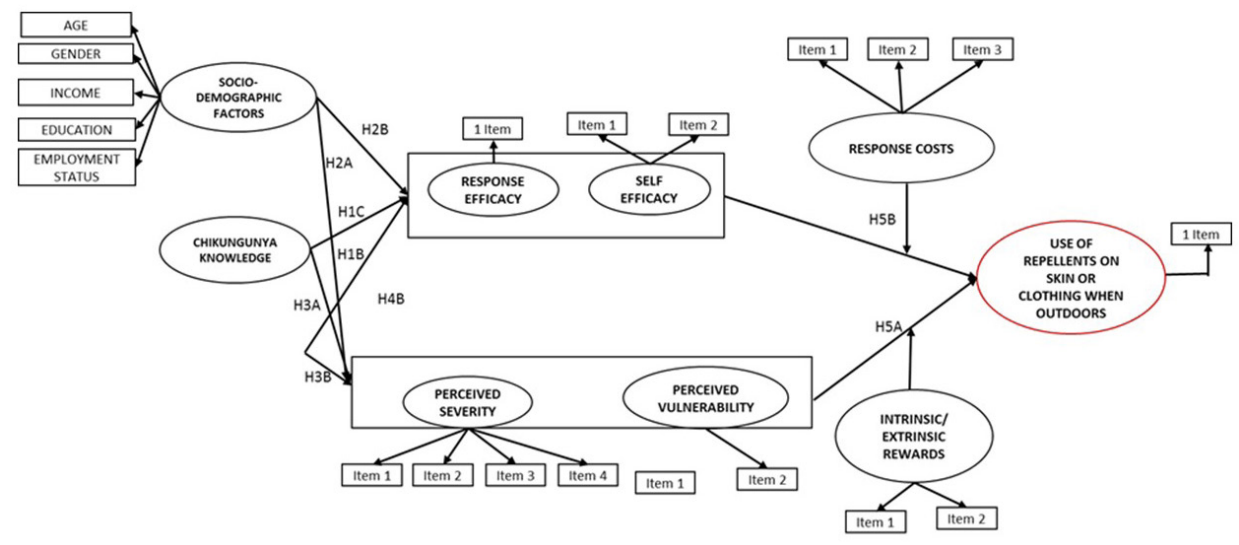

Figure 1. Conceptual Framework for the hypothesized Relationships Between Predictor Variables and Use of Insect Repellents Among US Travelers to Caribbean Destinations. 
repellents, and perceived self-efficacy for use of repellents on skin or clothing was investigated using the Pearson productmoment correlation coefficient (Table 1). Preliminary analyses were performed to ensure no violation of the assumptions of normality, linearity, or homoscedasticity. There was a significant positive correlation between Chikungunya knowledge and perceived severity $(r=0.32, \mathrm{n}=196, P<$ $0.001)$; Chikungunya knowledge and perceived vulnerability ( $r=0.22, \mathrm{n}=196, P=0.002)$; perceived severity and perceived vulnerability $(r=0.18, \mathrm{n}=196, P=0.013)$; perceived severity and perceived self-efficacy $(r=0.15, \mathrm{n}=196, \quad P=0.033)$; perceived vulnerability and perceived response efficacy $(r=0.57, \mathrm{n}=196, \quad P<0.001)$; perceived vulnerability and perceived self-efficacy $(r=0.26, \mathrm{n}=196, P<0.001)$, perceived response efficacy and perceived self-efficacy $(r=0.29, \mathrm{n}=196$, $P<0.001)$.

Ordinary least squares (OLS) regression was used to assess the ability of sociodemographic factors (age, gender, annual household income, and level of education) to predict levels of perceived Chikungunya severity. The prediction model was statistically significant, F $(9,174)=3.52, P<0.004$, and accounted for approximately $11 \%$ of the variance in perceived Chikungunya severity $\left(\mathrm{R}^{2}=0.15\right.$, adjusted $\left.\mathrm{R}^{2}=0.11\right)$. Each unit increase in age resulted in significantly lower scores on perceived Chikungunya severity (adjusted odd ratios $[\mathrm{AOR}]=0.02, \quad 95 \% \quad \mathrm{CI}=0.004-0.03, \quad P<0.040)$. Females had significantly lower perceived Chikungunya severity scores compared to males $(\mathrm{AOR}=0.28,95 \% \mathrm{CI}=0.07-0.49$, $P<0.010)$. Higher level of education was also associated with lower scores on perceived Chikungunya severity (Table 2). No significant relationship was found between income and respondents scores on perceived Chikungunya severity. OLS regression was also used to assess the ability of sociodemographic factors to predict levels of perceived vulnerability to Chikungunya. The prediction model was statistically significant, $F(9,174)=2.17, P<0.026$, and accounted for approximately $6 \%$ of the variance in perceived vulnerability to Chikungunya $\left(R^{2}=0.10\right.$, adjusted $\left.R^{2}=0.055\right)$. Each unit increase in age resulted in significantly lower scores on perceived vulnerability to Chikungunya $(\mathrm{AOR}=0.01$, 95\% CI $=0.002-0.03, P<0.029$ ). After adjusting for other variables in the model, increasing income was associated with significantly lower scores on perceived vulnerability to Chikungunya. No significant association was found between levels of education and scores on perceived vulnerability to Chikungunya $(P<0.05)$. OLS regression was also used to assess the ability of sociodemographic factors to predict levels of perceived response efficacy of use of insect repellents on skin and body as a Chikungunya PPM (Table 2). The prediction model was statistically significant, $\mathrm{F}(9,174)=3.84$, $P<0.001$, and accounted for approximately $12 \%$ of the variance in perceived response efficacy of insect repellents as a preventative behavior for Chikungunya infection $\left(R^{2}=0.17\right.$, adjusted $\mathrm{R}^{2}=0.12$ ). Increasing income and higher levels of education were also significantly correlated with higher scores on perceived response efficacy (Table 2). The prediction model for the relationship between sociodemographic factors and perceived self-efficacy for use of insect repellents was not statistically significant, $\mathrm{F}(9,174)=1.52, P=0.143)$, suggesting that age, gender, income, and level of education were not significant predictors of use of insect repellents among the study participants.

Direct logistic regression was performed to assess the impact of sociodemographic predictor variables on the likelihood that respondents will report use of insect repellents (dichotomous, $\mathrm{No}=0$, Yes $=1$ ) on skin or clothing when outdoors at a Caribbean destination. The full model containing all 4 sociodemographic predictors was statistically significant, $X^{2}(11,184)=32.04, P=0.001$, indicating that the model was able to distinguish respondents who reported from those who did not report use of insect repellents. The model as a whole explained between $16.0 \%$ (Cox and Snell $\mathrm{R}^{2}$ ) and $25.0 \%$ (Nagelkerke $\mathrm{R}^{2}$ ) of the variance in self-reported use of insect repellents. The results indicate that higher levels of education were associated with greater odds of self-reported use of insect repellents on skin or body when present at a Caribbean destination. For example, after adjusting for other variables in the model, the odds of self-reported use of insect repellents was $2 \mathrm{x}$ more for respondents with a 2-year college education when compared to respondents with only a high school education $(\mathrm{AOR}=2.1,95 \% \mathrm{CI}=1.42-6.03, P<0.001)$. Additionally, the odds of self-reported use of insect repellents was $3 \mathrm{x}$ that of respondents with only a high school education for respondents with a 4-year college degree $(\mathrm{AOR}=2.7,95 \%$ $\mathrm{CI}=2.10-8.29, P<0.001)$ or a graduate degree $(\mathrm{AOR}=2.8$, 95\% CI $=2.30-8.15)$.

Hierarchical binary logistic regression was conducted to assess the ability of perceived severity, perceived vulnerability, perceived response efficacy, perceived self-efficacy, and Chikungunya knowledge to predict self-reported use of insect repellents as a Chikungunya protective behavior, after controlling for age, gender, income, education. All cases

Table 1. Pearson Product-Moment Correlations Between Chikungunya Knowledge, Perceived Severity, Perceived Vulnerability, Response Efficacy, and Self-efficacy Respectively

\begin{tabular}{|c|c|c|c|c|c|c|}
\hline & & 1 & 2 & 3 & 4 & 5 \\
\hline 1. & Chikungunya knowledge & - & $0.32^{\mathrm{b}}$ & $0.22^{\mathrm{b}}$ & 0.11 & -0.01 \\
\hline 2. & Perceived severity & & - & $0.18^{\mathrm{a}}$ & 0.05 & $0.15^{\mathrm{a}}$ \\
\hline 3. & Perceived vulnerability & & & - & $0.57^{b}$ & $0.26^{b}$ \\
\hline 4. & Perceived response efficacy & & & & - & $0.29^{b}$ \\
\hline & Perceived self-efficacy & & & & & - \\
\hline
\end{tabular}

${ }^{a}$ Correlation is significant at the 0.05 level (2-tailed).

${ }^{\mathrm{b}}$ Correlation is significant at the 0.01 level (2-tailed). 


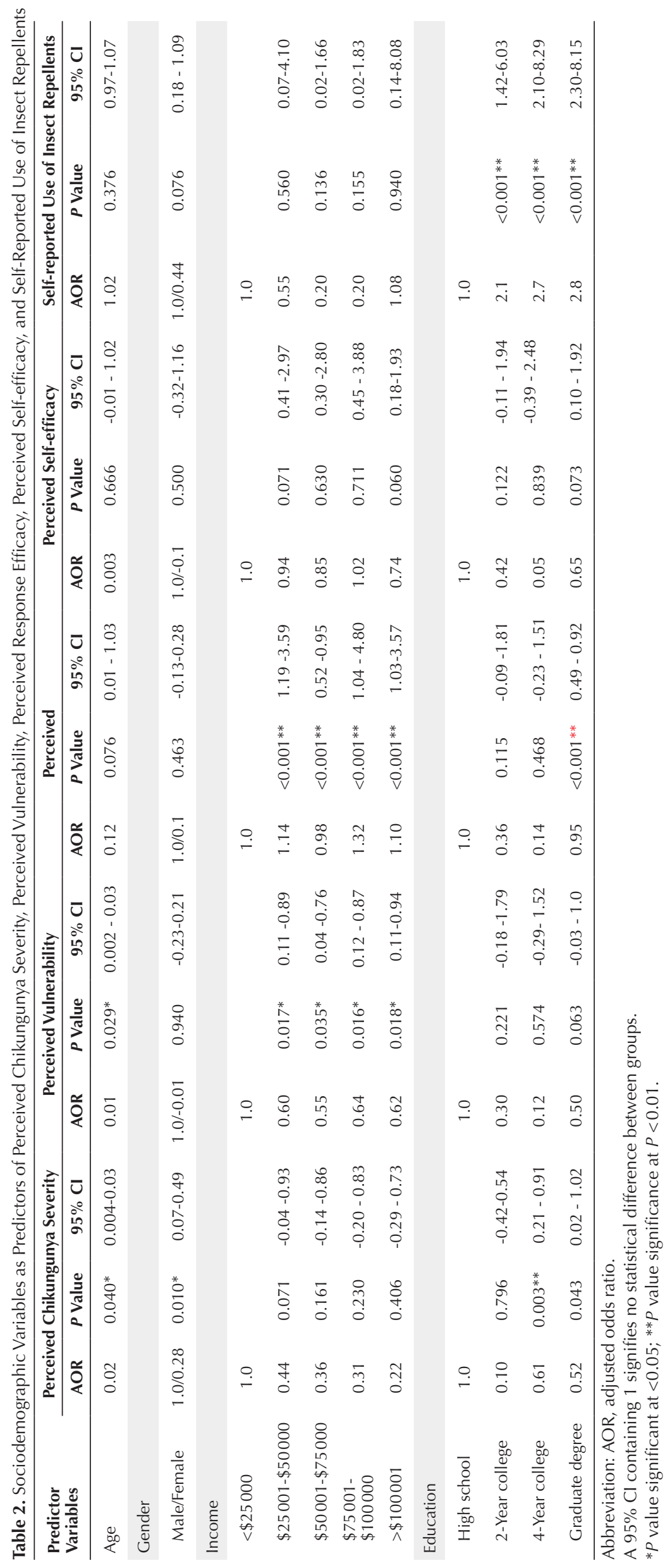


with missing values (6\%) were excluded from analysis. First, a baseline logistic regression model was run including all cases $(n=184)$ and requesting standardized residuals. Next, a revised logistic regression model was run, excluding outliers (standardized residual $\geq 2.58 ; \mathrm{n}=6$ cases $[3 \%]$ ). The model that excluded outliers was selected for interpretation, as it increased classification accuracy (91.6\%) compared to the baseline model's classification accuracy (86.4\%). Age, gender, income, employment status, and education were entered in step 1 , and together, they explained between $17 \%\left(\right.$ Cox \& Snell $\mathrm{R}^{2}=17.3$ ) and $28 \%$ (Nagelkerke $\mathrm{R}^{2}=28.3$ ) of variability in respondents use of insect repellents, $\chi^{2}(9,178)=33.77, P<0.001$. After entry of perceived severity, perceived vulnerability, perceived response efficacy, perceived self-efficacy, and Chikungunya knowledge in step 2, the total variance explained by the model as a whole was between $38 \%\left(\right.$ Cox $\&$ Snell $\left.\mathrm{R}^{2}=37.5\right)$ and $62 \%$ $\left(\right.$ Nagelkerke $\left.\mathrm{R}^{2}=61.5\right), \chi^{2}(14,178)=83.79, P<0.001$. After controlling for sociodemographic variables, the variables in step 2 explained between $20 \%$ (Cox \& Snell R ${ }^{2}=20.2$ ) and 33\% (Nagelkerke $\mathrm{R}^{2}=33.2$ ) of additional variability in respondents use of insect repellents, $\chi^{2}(5,178)=50.02, P<0.001$. The null hypothesis of no difference between the model with only the sociodemographic variables versus the model with the proximal Chikungunya-related predictor variables was therefore rejected. The existence of a hierarchical relationship between the more proximal Chikungunya-related variables and self-reported use of insect repellents as a PPM was supported (Table 3).

Among the proximal Chikungunya-related variables, perceived response efficacy, perceived self-efficacy and perceived severity were significantly associated with self- reported use of insect repellents. Adjusting for other variables in the model, each unit increase in respondent's scores on perceived response efficacy was associated with a $9 \mathrm{x}$ more likelihood of use of insect repellents $(\mathrm{AOR}=8.67$, $95 \% \mathrm{CI}=1.01-73.86, P=0.048)$. In the same vein, each unit increase in scores on perceived self-efficacy was associated with a $24 \mathrm{x}$ likelihood of use of insect repellents, after adjusting for other variables in the model $(\mathrm{AOR}=24.02,95 \% \mathrm{CI}=5.02$ - 114.99, $P<0.001)$. Holding other variables in the model constant, each unit increase in scores on perceived severity was associated with a $6 \mathrm{x}$ likelihood of use of insect repellents $(\mathrm{AOR}=6.40,95 \% \mathrm{CI}=2.01-20.40, P=0.002)$. No significant association was found between perceived vulnerability and Chikungunya knowledge, respectively, with self-reported use of insect repellents among the study participants $(P>0.05)$.

\section{Discussion}

Among the contributions of the present research is the exploration of the relationship between proximal Chikungunya-related variables and the use of insect repellents as a recommended PPM after controlling for the distal/sociodemographic variables. Study findings have important implications for health communication messaging aimed at reducing the spread of Chikungunya. The finding of significant positive correlation between the Chikungunya risk-related variables (i.e. Chikungunya knowledge, perceived severity, perceived vulnerability, perceived self-efficacy, and response efficacy respectively) supports the hypothesized relationships in Rogers' ${ }^{18}$ PMT, other psychological and behavioral theories, ${ }^{19}$ and previous research ${ }^{22}$ thus suggesting that heightening risk appraisals may have an effect on

Table 3. Hierarchical Multiple Logistic Regression Analysis - Predictors of Self-reported Use of Insect Repellents

\begin{tabular}{|c|c|c|c|c|c|c|}
\hline \multirow{3}{*}{ Predictor Variables } & \multicolumn{2}{|c|}{ Response Variable } & \multirow[b]{3}{*}{$95 \% \mathrm{Cl}$} & \multirow[b]{3}{*}{ AOR } & \multirow[b]{3}{*}{$P$ Value } & \multirow[b]{3}{*}{$95 \% \mathrm{Cl}$} \\
\hline & \multicolumn{2}{|c|}{ Self-reported Use of Insect Repellents } & & & & \\
\hline & UOR & $P$ Value & & & & \\
\hline Age & 0.96 & 0.055 & $0.92-1.00$ & 0.74 & $0.002^{* *}$ & $0.61-0.91$ \\
\hline \multicolumn{7}{|l|}{ Gender } \\
\hline Male & 1.0 & \multirow{2}{*}{$0.037^{*}$} & \multirow{2}{*}{$1.05-4.65$} & 1.0 & \multirow{2}{*}{$0.034^{*}$} & \multirow{2}{*}{$0.02-0.86$} \\
\hline Female & 2.21 & & & 0.32 & & \\
\hline \multicolumn{7}{|l|}{ Income } \\
\hline$<\$ 25000$ & 1.0 & & & 1.0 & & \\
\hline$\$ 25001-\$ 50000$ & 0.88 & 0.837 & $0.25-3.12$ & 0.03 & $0.044^{*}$ & $0.01-0.91$ \\
\hline$\$ 50001-\$ 75000$ & 2.13 & 0.286 & $0.53-8.48$ & 2.69 & 0.439 & $0.22-32.71$ \\
\hline$\$ 75001-\$ 100000$ & 1.83 & 0.466 & $0.36-9.35$ & 0.08 & 0.132 & $0.03-2.19$ \\
\hline$>\$ 100001$ & 0.35 & 0.112 & $0.09-1.28$ & 0.01 & $0.005^{*}$ & $0.00-0.24$ \\
\hline \multicolumn{7}{|l|}{ Education } \\
\hline High school & 1.0 & & & 1.0 & & \\
\hline 2-Year college & $0.00^{\mathrm{a}}$ & 0.999 & - & $0.00^{\mathrm{a}}$ & 0.999 & - \\
\hline 4-Year college & $0.00^{\mathrm{a}}$ & 0.999 & - & $0.00^{\mathrm{a}}$ & 0.999 & - \\
\hline Graduate degree & $0.00^{\mathrm{a}}$ & 0.999 & - & $0.00^{\mathrm{a}}$ & 0.999 & - \\
\hline Perceived response efficacy & 1.0 & 0.996 & $0.61-1.64$ & 8.67 & $0.048^{*}$ & $1.01-73.86$ \\
\hline Perceived self-efficacy & 2.09 & $0.002 * *$ & $1.31-3.34$ & 24.02 & $<0.000^{* * *}$ & $5.02-114.99$ \\
\hline Perceived severity & 1.69 & $0.031 *$ & $1.05-2.71$ & 6.40 & $0.002 * *$ & $2.01-20.40$ \\
\hline Perceived vulnerability & 0.95 & 0.829 & $0.57-1.56$ & 0.37 & 0.158 & $0.10-1.47$ \\
\hline Chikungunya knowledge & 1.05 & 0.435 & $0.93-1.19$ & 1.23 & 0.119 & $0.94-1.70$ \\
\hline
\end{tabular}

Abbreviation: UOR, unadjusted odds ratio; AOR, adjusted odds ratio.

a Evidence of multicollinearity (standard error $>2.0$ ), hence values not interpreted.

* $P$ value significance at $P<0.05 ; * * P$ value significance at $P<0.01 ; * * * P$ value significance at $P<0.001$. 
behavioral intentions and consequent health behavior. This study also reported a significant association between specific sociodemographic variables and some of the Chikungunya risk-related variables. For example, compared to males, females had significantly lower perceived Chikungunya severity scores. Each unit of increase in age was associated with significantly lower scores on perceived Chikungunya severity and on perceived vulnerability to Chikungunya. Higher levels of education were also associated with lower scores on perceived Chikungunya severity and higher scores on perceived response efficacy of use of insect repellents. Finally, higher income was associated with lower scores on perceived vulnerability to Chikungunya and higher scores on perceived response efficacy of use of insect repellents. Several studies have investigated the relationships between health beliefs and sociodemographic factors. ${ }^{16,39}$ These investigations were based on the premise that "Health decision-making is a process in which the individual moves through a series of stages," such that subsequent behavior is dependent on the interaction at each of these stages. ${ }^{20}$ Early evidence for the relationship between demographic factors, health beliefs, and health behavior was provided by the study of Kegeles et al. ${ }^{40}$ They reported that within all age, income, educational, and occupational groups, women who believed in the benefits of taking preventive action made the decision to take a Papanicolaou test for detecting cervical cancer more than women without those beliefs. ${ }^{40}$ Similar findings have been documented by other researchers. ${ }^{40}$ After analyzing the findings of several studies on use of health services, Rosenstock $^{20}$ concluded that relatively higher educated individuals were more likely to use health services. Lalani et $\mathrm{al}^{16}$ reported a significant association between the female gender and compliance with use of insect repellents. The current study found a significant association between higher levels of education and self-reported use of insect repellents for Chikungunya prevention. It is, therefore, reasonable to conclude that sociodemographic variables were significantly associated with the use of insect repellents among the study participants.

Beyond the joint effects of sociodemographic and psychosocial variables on preventative health behavior, this study tested whether psychosocial variables explained significant variability in the use of insect repellents beyond what is already explained by sociodemographic variables alone. Study results revealed the presence of a significant association between 3 of the proximal Chikungunya-related variables (perceived response efficacy, perceived selfefficacy, and perceived severity) and self-reported use of insect repellents on skin and clothing after controlling for the sociodemographic variables. No significant association was found between Chikungunya knowledge and selfreported use of insect repellents after controlling for other variables in the model. Several researchers have reported on the relationship between knowledge and risk-reduction behavior. Moro et $\mathrm{al}^{41}$ reported a positive association between knowledge of Chikungunya disease and future intentions to use mosquito repellent in Italy following the first reported outbreak of the disease in the northern hemisphere. Jepson et $\mathrm{al}^{42}$ reported that knowledge of cancer risk factors was a significant explanatory variable in the observed differences in cancer-preventive behavior between black and white racial groups in the United States. Although knowledge is important in behavior change, it is not sufficient to induce behavior change. Aspinwall et $\mathrm{al}^{43}$ reported no significant association between changes in sexual behavior at two time intervals and knowledge of HIV sero-status. No significant association was found between knowledge of breast cancer and breast selfexamination among women attending a breast clinic with control subjects. ${ }^{43}$

\section{Conclusion}

The current findings suggest that, after controlling for sociodemographic variables, an understanding of the levels of perceived response efficacy, perceived self-efficacy, and perceived severity among a representative sample of US travelers to Caribbean destinations has predictive value for self-reported use of insect repellents. The action-implications of the outcomes of this study seem quite clear. It seems essential to establish the use of insect repellents on skin and clothing as a Chikungunya disease personal preventive measure within an educational context, framed along the lines of Chikungunya severity, response efficacy, and self-efficacy, during occasions where US travelers are at destinations with a high risk of exposure to Chikungunya disease-carrying mosquitoes. Public health officials need to increase Chikungunya disease awareness among US travelers to Caribbean destinations. Such awareness should include information on the severity of Chikungunya in relation to personal health, employment, and family relationships. The benefits of taking preventative action, including the correct procedures to effectively use insect repellents on skin and clothing, should be advocated.

\section{Study Limitations}

The associations modeled in this study were based on selfreporting. It remains to be seen whether self-reporting mirrors actual patterns of use of insect repellents on skin or body when outdoors at a Caribbean destination. For example, the observed significant association between higher levels of education and use of insect repellents may be a true pattern, suggesting that higher education results in the greater likelihood of use of insect repellents. On the contrary, this association could be attributed to greater articulation of this behavior as desirable by the more educated respondents, in which case they will more likely self-report engaging in the said behavior. A suggestion for minimizing this discrepancy would be to design a study in which participants would be observed/recruited at the destination, and records made of actual adoption of the recommended PPM. This would also minimize the bias due to recall and self-reporting.

The proportion of respondents who reported using insect repellents on skin and clothing was more than twice those who did not report use of insect repellents on skin and clothing. It is quite possible that the results of this study may be impacted by this uneven representation. Future studies can limit this impact by oversampling. Sample size limitations made it impossible to implement a model containing the 
full range of variables in the PMT. For example, interaction terms between response-/self-efficacy, perceived severity/ perceived vulnerability, respectively, could not be included in the model for this reason. It is expected that future studies with a larger sample size will be able to test their effect on the use of insect repellents among study participants. In spite of these limitations, the findings of this study have important implications on planning programs for Chikungunya riskreduction among U.S. travelers to Caribbean destinations. The findings also reveal that the protection motivation theory is of practical relevance for investigating the predictors of use of insect repellents on skin or clothing as a PPM against Chikungunya infection. Nonetheless, future studies should aim at building on the limitations and findings from this study.

\section{Authors' Contributions}

All listed authors contributed substantially to the completion of this manuscript.

\section{Conflict of Interest Disclosures \\ No conflict of interest.}

\section{Ethical Approval}

The authors have addressed the relevant ethical issues. Informed consent was obtained from all participants before data collection, and the research protocol was approved by the University of Florida Institutional Review Board (IRB\#: 2015-U-1040).

\section{Funding/Support}

This study received no funding or external support.

\section{References}

1. Evans SJ. Thousands of holidaymakers return from Caribbean with deadly mosquito virus that Lindsay Lohan was 'hospitalized' with. http://www.dailymail.co.uk/news/article-2942957/Thousandsholidaymakers-return-Caribbean-deadly-mosquito-virus-LindsayLohan-hospitalized-with.html\#ixzz3lclQ7gwY. Accessed October 6, 2016. Published 2015.

2. Britain warns travellers to the Caribbean about Chikungunya virus. http://www.jamaicaobserver.com/news/Britain-warns-travellersto-the-Caribbean-about-Chikungunya-virus. Accessed October 9, 2016. Published 2015.

3. Pearson E. Nearly 700 New Yorkers caught chikungunya virus last year after travelling to Caribbean or other tropical locales. http://www.nydailynews.com/life-style/health/mosquito-bornechikungunya-virus-sickening-travelers-article-1.2105584. Accessed October 6, 2016. Published 2015.

4. Leparc-Goffart I, Nougairede A, Cassadou S, Prat C, de Lamballerie X. Chikungunya in the Americas. Lancet. 2014;383(9916):514. doi:10.1016/s0140-6736(14)60185-9.

5. Chikungunya fever diagnosed among international travelers-United States, 2005-2006. MMWR Morb Mortal Wkly Rep. 2006;55(38):1040-1042.

6. Sharp TM, Roth NM, Torres J, et al. Chikungunya cases identified through passive surveillance and household investigations--Puerto Rico, May 5-August 12, 2014. MMWR Morb Mortal Wkly Rep. 2014;63(48):1121-1128.

7. Kendrick K, Stanek D, Blackmore C. Notes from the field: Transmission of chikungunya virus in the continental United States-Florida, 2014. MMWR Morb Mortal Wkly Rep. 2014;63(48):1137.

\section{Research Highlights}

\section{What Is Already Known?}

The relationship between international travel and the global spread of Chikungunya disease is well-established. Although 'use of insect repellents on skin and clothing' has been known to be effective for Chikungunya disease prevention, its sociodemographic and behavioral correlates, particularly among US travelers to Caribbean destinations has remained largely understudied.

\section{What This Study Adds?}

Study findings suggest that after controlling for sociodemographic variables, an understanding of the levels of perceived response efficacy, perceived self-efficacy, and perceived severity among a representative sample of US travelers to Caribbean destinations has predictive value for self-reported use of insect repellents on skin and clothing.

8. Kuehn BM. Chikungunya virus transmission found in the United States: US health authorities brace for wider spread. JAMA. 2014;312(8):776-777. doi:10.1001/jama.2014.9916.

9. Roche B, Gaillard B, Leger L, et al. How human behavior drives the propagation of an emerging infection: the case of the 2014 Chikungunya outbreak in Martinique. bioRxiv. 2016. doi:10.1101/052183

10. US Centers for Disease Control Prevention. Chikungunya in the Caribbean. http://wwwnc.cdc.gov/travel/notices/watch/ chikungunya-caribbean. Accessed October 6, 2016. Published March 2015.

11. Nasci RS, Zielinski-Gutierrez E, Wirtz RA, Brogdon WG. Protection against mosquitoes, ticks, \& other insects \& arthropods. CDC Health Information for International Travel 2014: The Yellow Book. 2013;56(2):94.

12. US Army Public Health Center. DoD Insect Repellent System. https://phc.amedd.army.mil/topics/envirohealth/epm/Pages/ DoDInsectRepellentSystem.aspx. Accessed October 4, 2016. Published 2016.

13. Fradin MS, Day JF. Comparative efficacy of insect repellents against mosquito bites. N Engl J Med. 2002;347(1):13-18. doi:10.1056/ NEJMoa011699.

14. Omodior O, Pennington-Gray L, Holland S, Thapa B, Kiousis S. Chikungunya disease awareness among U.S. travelers to Caribbean destinations. Int J Travel Med Glob Health. 2017;5(1):20-27. doi:10.15171/ijtmgh.2017.04

15. Millman AJ, Esposito DH, Biggs HM, et al. Chikungunya and dengue virus infections among United States community service volunteers returning from the Dominican Republic, 2014. Am JTrop Med Hyg. 2016;94(6):1336-1341. doi:10.4269/ajtmh.15-0815.

16. Lalani T, Yun H, Tribble D, et al. A comparison of compliance rates with anti-vectorial protective measures during travel to regions with dengue or chikungunya activity, and regions endemic for Plasmodium falciparum malaria. J Travel Med. 2016;23(5). doi:10.1093/jtm/taw043.

17. Dehecq JS, Baville M, Margueron T, Mussard R, Filleul L. The reemergence of the Chikungunya virus in Reunion Island on 2010: evolution of the mosquito control practices. Bull Soc Pathol Exot. 2011;104(2):153-160. doi:10.1007/s13149-010-0121-5.

18. Rogers R. Cognitive and physiological processes in fear appeals and attitude change: A revised theory of protection motivation. In: Cacioppo DJ, Petty R, eds. Social Psychophysiology. New York: Guildford Press; 1983:153-176.

19. Janz NK, Becker MH. The health belief model: a decade later. Health Educ Q. 1984;11(1):1-47. doi:10.1177/109019818401100101.

20. Rosenstock IM. Why people use health services. Milbank Q. 
2005;83(4). doi:10.1111/j.1468-0009.2005.00425.x.

21. Gerrard M, Gibbons FX, Bushman BJ. Relation between perceived vulnerability to HIV and precautionary sexual behavior. Psychol Bull. 1996;119(3):390-409. doi:10.1037/0033-2909.119.3.390.

22. Sheeran P, Harris PR, Epton T. Does heightening risk appraisals change people's intentions and behavior? A meta-analysis of experimental studies. Psychol Bull. 2014;140(2):511-543. doi:10.1037/a0033065.

23. Chen JY, Fox SA, Cantrell CH, Stockdale SE, Kagawa-Singer M. Health disparities and prevention: racial/ethnic barriers to flu vaccinations. J Community Health. 2007;32(1):5-20.

24. Hyman RB, Baker S, Ephraim R, Moadel A, Philip J. Health Belief Model variables as predictors of screening mammography utilization. J Behav Med. 1994;17(4):391-406. doi:10.1007/ BF01858010.

25. Bandura A. Self-efficacy: toward a unifying theory of behavioral change. Psychol Rev. 1977;84(2):191-215.

26. Floyd DL, Prentice-Dunn S, Rogers RW. A meta-analysis of research on protection motivation theory. J Appl Soc Psychol. 2000;30(2):407-429. doi:10.1111/j.1559-1816.2000.tb02323.x.

27. Jessor R, Turbin MS, Costa FM. Protective factors in adolescent health behavior. J Pers Soc Psychol. 1998;75(3):788-800. doi:10.1037/0278-6133.13.1.86

28. Boer H, Seydel ER. Protection Motivation Theory. Philadelphia: Open University Press; 1996.

29. Milne $S$, Sheeran $P$, Orbell S. Prediction and intervention in health-related behavior: a meta-analytic review of protection motivation theory. J Appl Soc Psychol. 2000;30(1):106-143. doi:10.1111/j.1559-1816.2000.tb02308.x.

30. Jones JL, Leary MR. Effects of appearance-based admonitions against sun exposure on tanning intentions in young adults. Health Psychol. 1994;13(1):86-90. doi:10.1037/0278-6133.13.1.86.

31. Wulfert E, Wan CK. Condom use: a self-efficacy model. Health Psychol. 1993;12(5):346-353. doi:10.1037/0278-6133.12.5.346.

32. Kaiser HF. An index of factorial simplicity. Psychometrika. 1974;39(1):31-36. doi:10.1007/bf02291575.
33. Pallant J. SPSS Survival Manual: A Step by Step Guide to Data Analysis Using SPSS. Maidenhead: Open University Press/ McGraw-Hill; 2010.

34. U.S. Centers for Disease Control and Prevention. Chikungunya. https://wwwnc.cdc.gov/travel/diseases/Chikungunya. Accessed March 16, 2017. Published 2013.

35. Rippetoe PA, Rogers RW. Effects of components of protectionmotivation theory on adaptive and maladaptive coping with a health threat. J Pers Soc Psychol. 1987;52(3):596-604. doi:10.1037/0022-3514.52.3.596.

36. Ho R. Predicting intention for protective health behaviour: A test of the protection versus the ordered protection motivation model. Aust J Psychol. 2000;52(2):110-118. doi:10.1080/00049530008255376.

37. Umeh K. Cognitive appraisals, maladaptive coping, and past behaviour in protection motivation. Psychol Health. 2004;19(6):719-735. doi:10.1080/0887044042000196692.

38. Galanos AN, Strauss RP, Pieper CF. Sociodemographic correlates of health beliefs among black and white community dwelling elderly individuals. Int J Aging Hum Dev. 1994;38(4):339-350. doi:10.2190/62ka-fwn1-6xv5-pr2q.

39. Kegeles SS, Kirscht JP, Haefner DP, Rosenstock IM. Survey of beliefs about cancer detection and taking Papanicolaou tests. Public Health Rep. 1965;80(9):815-823. doi:10.2307/4592543.

40. Moro ML, Gagliotti C, Silvi G, et al. Knowledge, attitudes and practices survey after an outbreak of chikungunya infections. Int Health. 2010;2(3):223-227. doi:10.1016/j.inhe.2010.07.003.

41. Jepson C, Kessler LG, Portnoy B, Gibbs T. Black-white differences in cancer prevention knowledge and behavior. Am J Public Health. 1991;81(4):501-504. doi:10.2105/AJPH.81.4.501.

42. Aspinwall LG, Kemeny ME, Taylor SE, Schneider SG, Dudley JP. Psychosocial predictors of gay men's AIDS risk-reduction behavior. Health Psychol. 1991;10(6):432-444. doi:10.1037/02786133.10.6.432.

43. Owens RG, Daly J, Heron K, Leinster SJ. Psychological and social characteristics of attenders for breast screening. Psychol Health. 1987;1(4):303-313. doi:10.1080/08870448708400333. 